\title{
Obstructive Lung Disease in HIV—Phenotypes and Pathogenesis
}

\author{
Deepti Singhvi $^{1} \cdot$ Jessica Bon ${ }^{1,2} \cdot$ Alison Morris $^{1}$
}

Published online: 29 June 2019

(C) Springer Science+Business Media, LLC, part of Springer Nature 2019

\begin{abstract}
Purpose of Review In the antiretroviral therapy era, people living with HIV (PLWH) are surviving to older ages. Chronic illnesses such as chronic obstructive pulmonary disease (COPD) occur more frequently. COPD is often described as a single entity, yet multiple manifestations may be considered phenotypes. HIV is an independent risk factor for certain COPD phenotypes, and mechanisms underlying pathogenesis of these phenotypes may differ and impact response to therapy.

Recent Findings Impaired diffusing capacity, airflow obstruction, and radiographic emphysema occur in PLWH and are associated with increased mortality. Age, sex, tobacco, and HIV-specific factors likely modulate the severity of disease. An altered lung microbiome and residual HIV in the lung may also influence phenotypes.

Summary COPD is prevalent in PLWH with multiple phenotypes contributing to the burden of disease. HIV-specific factors and the respiratory microbiome influence disease pathogenesis. As tobacco use remains a significant risk factor for COPD, smoking cessation must be emphasized for all PLWH.
\end{abstract}

Keywords Chronic obstructive $\cdot \mathrm{HIV} \cdot$ Pulmonary $\cdot$ Emphysema $\cdot$ Phenotypes $\cdot$ Pathogenesis

\section{Introduction}

Human immunodeficiency virus (HIV) is a global disease that affects more than 36 million people worldwide [1]. With the advent of antiretroviral therapy (ART), life expectancy for people living with HIV (PLWH) is approaching that of individuals without HIV. While the major causes of pulmonary complications in PLWH were infectious diseases in the preART era, the prevalence of noninfectious pulmonary diseases has increased since the implementation of ART. As PLWH live to older ages, chronic lung diseases have begun to emerge. These noninfectious diseases often occur at younger ages than in HIV-uninfected individuals and cases are rising, especially in the 30-49-year-old age group [2].

This article is part of the Topical Collection on HIV Pathogenesis and Treatment

Deepti Singhvi

singhvidg@upmc.edu

1 Department of Medicine, University of Pittsburgh, Pittsburgh, Pennsylvania, USA

2 VA Pittsburgh Healthcare System, Pittsburgh, Pennsylvania, USA
Chronic obstructive pulmonary disease (COPD) is common in PLWH, with HIV infection increasingly being recognized as an independent COPD risk factor $[3 \cdot 4-8]$. COPD is characterized by persistent respiratory symptoms and airflow obstruction that is not fully reversible and defined by the Global Initiative for Chronic Obstructive Lung Disease (GOLD) criteria as a forced expiratory volume in $1 \mathrm{~s}$ to forced vital capacity (FEV1/FVC) ratio less than 0.7 or below the 5 th percentile (lower limit of normal (LLN)) on spirometry [9]. While the presence of airflow limitation is diagnostic of COPD, COPD is well-recognized as a complex syndrome, rather than a single disease, with both pulmonary and extrapulmonary manifestations. Significant heterogeneity exists among affected individuals with respect to symptoms, lung function, radiographic imaging, response to therapy, and survival. As such, collections of common elements are often subgrouped into clinical COPD phenotypes, previously defined as "a single or combination of disease attributes that describe differences between individuals with COPD as they relate to clinically meaningful outcomes" [10]. The goal of phenotyping is to identify groups of individuals who share prognostic or therapeutic features that could help guide management. Many of these COPD phenotypes are unique to PLWH. Given the significant burden of chronic lung disease 
in PLWH and its associated impact on mortality, we review the myriad phenotypes of COPD in PLWH. We will also focus on the role of HIV in the lung and the lung microbiome, key mechanisms of interest in the complex pathogenesis of COPD in PLWH.

\section{Phenotypes of Obstructive Lung Disease}

\section{Airflow Obstruction}

COPD, as defined by GOLD criteria with fixed airflow obstruction, is common in PLWH with prevalence estimates ranging from 6 to $21 \%\left[8,11-15^{\circ}\right]$. This wide range is likely due to differences in the characteristics of different cohorts and limited by the cross-sectional, single-center nature of most studies. For example, two cross-sectional studies of PLWH in the United States (USA) found widely varying prevalences of obstruction. George et al. found $6.8 \%$ of PLWH to meet a criterion of FEV1/FVC ratio $<0.7$ ( $8.6 \%$ by LLN), whereas a later study in a different US location identified $21 \%$ of participants to have an FEV1/FVC ratio $<0.7$ (19\% by LLN) [12, 13]. Participants in the former study were younger, and the cohort contained a lower percentage of smokers and intravenous drug users, and a significantly smaller fraction of individuals with a history of Pneumocystis pneumonia or hepatitis $\mathrm{C}$ infection, making the lower prevalence of COPD potentially due to a baseline healthier population with fewer risk factors for airflow obstruction.

There are multiple risk factors for obstruction in PLWH. Lower FEV1/FVC ratio has been associated with increasing age, and severity of obstruction is independently associated with pack-year smoking history $[8,11-13,15 \cdot, 16]$. One recent study of PLWH in Copenhagen, however, found no association between cumulative smoking history and severity of airflow limitation [14]. This population had a large number of never-smokers, which may have reduced the power of the study to find associations with smoking. Other studies have shown associations between worsening airflow limitation and prior TB infection, history of bacterial or Pneumocystis pneumonia, ART use, HIV RNA > 75,000 copies/mL, intravenous drug use, higher GOLD stage, and male sex [6, 11-13, $16,17 \cdot \bullet, 18]$.

Rates of decline in FEV1 have been well-studied in HIVuninfected individuals with COPD, but fewer longitudinal studies have been conducted in PLWH. In the HIVuninfected population, annual decline in FEV1 for individuals with COPD can range from -20 to $-80 \mathrm{~mL}$ and varies depending on smoking status, disease susceptibility, and severity of disease at baseline $[19,20]$. In PLWH who smoke, baseline FEV1 and FVC are significantly lower than in uninfected smokers [6, 14], but whether the annual rate of decline in FEV1 differs between PLWH and uninfected smokers has not been clearly established. Active smokers have a faster decline in lung function compared to former and lifetime nonsmokers. Li et al. found that smoking 10-19.9 pack years had a small, but not statistically significant association with rapid FEV1 decline (defined as $>80 \mathrm{~mL} /$ year) [17••]. In a much larger multi-center study, MacDonald et al. used data from the Strategic Timing of Antiretroviral Treatment (START) Pulmonary Substudy to show a more rapid FEV1 decline in smokers $(-38.3 \mathrm{~mL} /$ year) than nonsmokers $(-25.1 \mathrm{~mL} /$ year $)$, a difference similar to that attributed to smoking in HIVuninfected cohorts [21•]. The high prevalence of airflow obstruction in PLWH along with accelerated lung function decline attributed to smoking support a need to emphasize tobacco cessation in PLWH.

Other risk factors for decline in FEV1 include older age, male sex, marijuana use, and worse baseline lung function $[17 \cdot \bullet]$. In a longitudinal analysis of intravenous drug users, Drummond et al. demonstrated an average decrease in FEV1 of $-35.8 \mathrm{~mL} /$ year in PLWH compared to a decline of $23.6 \mathrm{~mL} /$ year in HIV-uninfected participants $(p=0.135)$. In a subgroup analysis of this study, PLWH with HIV RNA > 75,000 copies $/ \mathrm{mL}$ had a significantly greater annual decline in FEV1 (- $99.1 \mathrm{~mL} /$ year) than HIV-uninfected individuals ($23.5 \mathrm{~mL} /$ year). These findings have not been reproduced, as the majority of studies comparing these two populations headto-head have been cross-sectional analyses and few individuals have high viral levels. However, recent longitudinal studies in only PLWH demonstrated decreases in FEV1 of $62 \mathrm{~mL} /$ year [17•.0 and a range of -22.2 to $-32.9 \mathrm{~mL} /$ year depending on baseline smoking status and timing of ART initiation $[22 \cdot \bullet]$, rates that are comparable to estimates in HIV-uninfected individuals with COPD.

\section{Low DLCO}

The most common pulmonary function abnormality in HIV is a reduction in diffusing capacity of the lungs for carbon monoxide (DLCO) [23, 24]. DLCO measures the ability of the lungs to transfer gas from inhaled air to red blood cells within pulmonary capillaries. An impairment in diffusing capacity suggests a defect within the alveolar-capillary membrane or problems with capillary blood volume or hemoglobin content. Emphysema is one of the most common causes of reduced DLCO and often associated with airway obstruction; therefore, reduced DLCO is often considered a phenotype of COPD. HIV is an independent predictor of decreased DLCO, with prevalence estimates in PLWH ranging from 53 to $84 \%$ depending on the population studied $[11,12,17 \bullet \cdot, 25]$. HIV-infected individuals with impaired diffusion are more likely to report respiratory symptoms, including cough, sputum production, and dyspnea [23, 24].

Risk factors for diffusion impairment are similar to those in HIV-uninfected individuals, but also include several factors 
that are unique to PLWH. Current and former smoking are well known to be associated with deficits in DLCO in the general population as well as in PLWH $[11,26]$. In PLWH, prior bacterial or Pneumocystis pneumonia, cocaine use, low body mass index (BMI), and advanced HIV infection are associated with lower DLCO $[11,24]$. DLCO reduction has been identified in nonsmokers with HIV as well, with prevalence estimates of impaired DLCO ranging from 9 to $48 \%$ in US cohorts [12, 23, 25]. Diffusion impairment is also associated with pulmonary vascular disease, airflow obstruction, and airway inflammation. Data on the progression of DLCO in PLWH are limited, but a small study showed that DLCO remained relatively constant over 6 months [27].

\section{Radiographic Emphysema}

Emphysema, characterized by enlargement of terminal air sacs and destruction of alveolar walls, occurs in PLWH. Emphysema was prevalent in the pre-ART era, with one study showing a prevalence of $15 \%$ in PLWH compared to $2 \%$ in HIV-uninfected controls [28]. Data from the ART era have shown even greater prevalence in PLWH with estimates ranging from 25 to $41 \%$, likely in part due to longer life expectancies allowing more time for PLWH to develop emphysema $[11,29,30,31 \bullet \bullet, 32 \bullet \bullet, 33]$. Variation in these estimates may be due to reliance on the use of semi-quantitative versus qualitative (with more inter-reader variability) methods and variation in thresholds used for the determination of the presence versus absence of emphysema. Despite the variability in prevalence estimates, these studies have established that radiographic emphysema is common in PLWH and have shown associations between emphysema and impaired physical activity, smoking exposure, increased age, and lower BMI $[11,33]$.

Recent studies have focused on predictors of emphysema progression. In one longitudinal study of 345 PLWH treated with ART, $41 \%$ had emphysema at baseline with $17.4 \%$ of participants showing progression of emphysema at a median follow-up time of 3.93 years [32••]. In this cohort, those who progressed had higher baseline emphysema scores, higher cumulative smoking histories, and more IV drug use. Emphysema progression was also related to DLCO \%predicted and FEV1/FVC ratio at baseline. The authors were able to predict emphysema progression over a 7-year period using a prediction model that included DLCO and emphysema distribution (area under the curve statistic of 0.85), suggesting a potential role for this tool in clinical practice. Another study demonstrated a negative correlation between plasma $\mathrm{CD}^{+} /$ $\mathrm{CD}^{+}$ratios and severity of radiographic emphysema that was independent of smoking, age, and HIV-related factors [31••]. The authors posit that this decreased ratio in peripheral blood may reflect a state of chronic immune activation and inflammation that could relate to the pathogenesis of emphysema itself. While causation cannot be established in a cross- sectional study, these findings suggest that a plasma test may serve as a potential marker of emphysema severity in PLWH.

\section{Overlap of COPD with Other Comorbidities}

COPD has been associated with multiple comorbidities including cardiovascular disease, diabetes, osteoporosis, and psychological disorders [34-36]. In PLWH, COPD has also been associated with these extrapulmonary comorbidities, which often present at a younger age than in uninfected individuals. As these comorbidities, including frailty, osteoporosis, cardiovascular disease, and pulmonary hypertension, can negatively impact both quality of life and overall prognosis, early recognition and management are essential.

\section{Frailty}

Frailty, a clinical syndrome of decreased reserve and increased vulnerability to stressors, is associated with increased age and leads to decreased physical function. As PLWH live to older ages, frailty has become increasingly prevalent, often at younger ages than in the HIV-uninfected population [37], and HIV is independently associated with frailty in middle-aged PLWH compared to uninfected controls [38]. Frailty is known to be associated with COPD as well, and this association is modified by the presence of concurrent HIV. A study of frailty in the Veterans Aging Cohort Study (VACS) cohort identified COPD as independently associated with frailty in both PLWH and HIV-uninfected participants, though the association was greater in PLWH [39•]. The combination of both COPD and HIV significantly increases frailty, perhaps due to the role of inflammation in both diseases. Thus, optimizing COPD management should be an important priority to minimize frailty and may help maintain physical function for aging PLWH.

\section{Osteoporosis}

COPD and emphysema are associated with low bone mineral density (BMD) and increased fracture risk [35, 40]. Low BMD and increased fracture risk are also more likely in PLWH compared to the general population [41, 42]. A recent study examining the combined effects of emphysema and HIV in a cohort of PLWH with radiographic chest CT imaging found that greater quantitative emphysema was independently associated with decreased BMD as estimated by thoracic vertebral bone attenuation in PLWH [43•]. The use of ART was also associated with decreased BMD compared to PLWH who were not on ART. Additional work is needed to determine if PLWH who have COPD should have more frequent screening for osteoporosis. 


\section{Cardiovascular Disease}

Coronary artery disease (CAD) is known to be a common comorbidity in PLWH, with significant attendant risk of mortality $[44,45]$. The development of early cardiovascular disease in PLWH may be related to the pathogenesis of COPD with both diseases linked to a chronic state of inflammation. Early detection of subclinical CAD is paramount to limiting complications from cardiovascular disease, and the use of coronary artery calcium (CAC) scores obtained from chest computed tomography (CT) imaging has been validated as a method of estimating CAD. One study demonstrated that estimates of CAC obtained through traditional CT chest radiography correlate well to the traditional electrocardiogram (EKG)-cardiac CT in PLWH [46]. As chest imaging is often obtained in PLWH for alternate indications, such as evaluation of emphysema, the use of non-EKG gated CT scans for estimating CAC scores could improve the ease with which subclinical CAD can be identified in this high-risk population. The frequent coexistence of these two findings was demonstrated in a recent large observational study of asymptomatic PLWH on ART in which CAC scores and qualitative estimates of emphysema were calculated from CT chest images [47•]. There were significantly higher emphysema scores in participants with elevated CAC scores, and the presence of any emphysema in current smokers was independently associated with a positive CAC score. This cohort was approximately 20 years younger than the general HIV-uninfected population with both emphysema and $\mathrm{CAC}$, again demonstrating that chronic comorbidities often affect PLWH at younger ages.

\section{Pulmonary Hypertension}

Pulmonary hypertension (PH) is prevalent in PLWH and also appears to be more common in PLWH who have COPD. The link between COPD and PH in PLWH was first established in a cross-sectional analysis of outpatient PLWH in whom pulmonary function testing and transthoracic echocardiography (TTE) were obtained [48]. In this group, $15.5 \%$ of participants had an elevated pulmonary artery systolic pressures (PASP > $40 \mathrm{mmHg}$ ) and $7.8 \%$ had an elevated tricuspid regurgitant velocity (TRV). The presence of elevated PASP and TRV were significantly associated with both airflow obstruction and decreased DLCO. Increased PASP and TRV were also associated with elevated levels of markers of pulmonary and peripheral inflammation. A more recent study investigating participants from the VACS cohort who were referred for TTE found that $28 \%$ of PLWH had elevated PASP and that these individuals were more likely to have higher viral loads, lower $\mathrm{CD} 4^{+}$counts, and less likely to have had exposure to ART compared to PLWH with normal PASP [49]. There was a greater prevalence of COPD in PLWH with PH compared to PLWH without $\mathrm{PH}$. These findings suggest that $\mathrm{PH}$ and
COPD share a common underlying mechanism in PLWH and that perhaps both diseases are driven by a state of immune activation and persistent inflammation.

\section{COPD Phenotypes and Mortality}

The decreased lung function observed in PLWH, along with specific COPD phenotypes, has been linked to increased mortality. One recent study compared the VACS Index, a validated tool that predicts mortality in PLWH, between individuals with and without HIV infection in a multicenter Veterans Affairs (VA) Medical Center population [50••]. The authors found a significantly higher VACS Index in PLWH compared to HIV-uninfected controls. They also identified an association between mortality and airflow obstruction, impaired DLCO, and radiographic emphysema in PLWH that was independent of smoking history or VACS index. Similarly, another recent study showed increased mortality in PLWH who had an FEV1/FVC ratio less than 0.7 or DLCO $<60 \%$ predicted compared to PLWH with normal lung function, independent of smoking history [51••]. Kaplan-Meier curves in this study diverged from normal sooner in those with a DLCO $<60 \%$ predicted than in those with FEV1/FVC $<0.7$, suggesting that diffusion impairment may be an earlier marker of mortality. Many of those found to have obstruction in this study had no formal diagnosis of COPD. Finally, in a study of the VACS cohort investigating PH in PLWH compared to uninfected controls, PLWH with PH had the highest mortality. This mortality risk increased with rising PASP and was more significant at a lower PASP value in PLWH compared to uninfected participants. These data demonstrate that a variety of COPD phenotypes in PLWH are linked to increased mortality, highlighting the importance of regular screening in order to detect pulmonary and cardiovascular abnormalities in this population.

\section{Pathogenesis of Obstructive Lung Disease}

While smoking history explains the pathogenesis of most obstructive lung disease in developed countries, the pathogenesis of COPD in PLWH may also be independent of tobacco exposure $[3 \cdot, 4-8]$. The underlying mechanisms by which COPD develops in PLWH, independent of smoking-related changes, remain unclear. The pathogenesis of obstructive lung disease in this population is likely multifactorial (Fig. 1), with a large component attributable to a state of chronic inflammation in the setting of latent HIV infection [7, 52-54]. The effects of HIV viral load, reduced $\mathrm{CD} 4^{+} \mathrm{T}$ lymphocyte count, ART exposure, residual HIV in the lung, chronic inflammation, and an altered lung microbiome likely all play a role in the development of this highly prevalent disease. 
Fig. 1 Potential mechanisms for COPD pathogenesis in people living with HIV

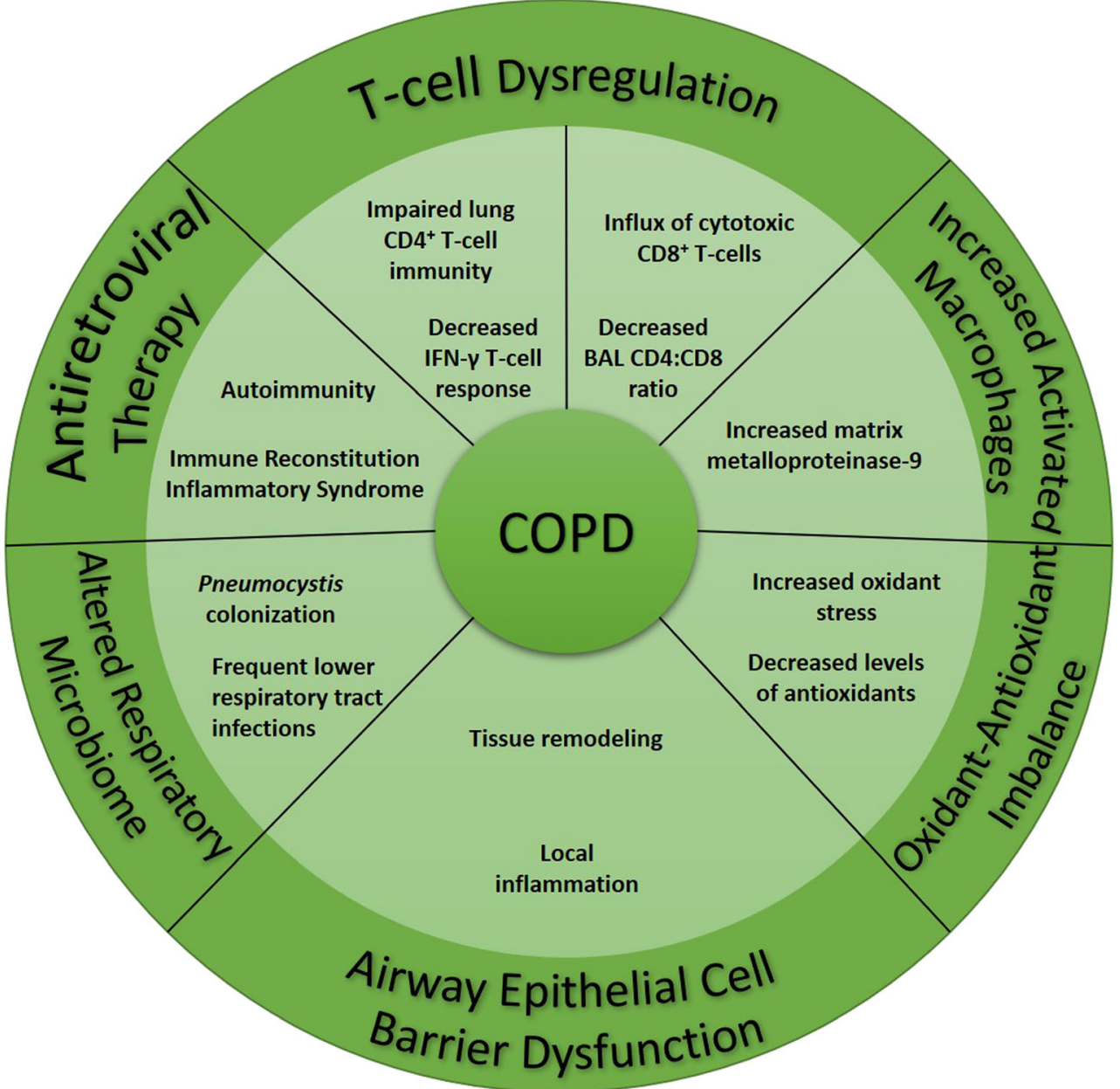

\section{Effect of HIV-Specific Factors}

The contributions of HIV-related factors such as viral load, $\mathrm{CD}^{+}{ }^{+} \mathrm{T}$ lymphocyte count, and ART to the pathogenesis and progression of obstructive lung disease have been debated, with contradictory findings from different populations with varying exposures. In the pre-ART era, it was welldescribed that acquired immune deficiency syndrome (AIDS) was associated with emphysema and reduced DLCO $[55,56]$. Immune reconstitution that occurs with ART may reverse or prevent the pulmonary complications of advanced HIV, but data are conflicting. Below, we review the impact of HIV-associated variables on pulmonary function in PLWH.

\section{CD4 ${ }^{+}$T Lymphocyte Count}

Numerous studies in the ART era have investigated the role of $\mathrm{CD}^{+}$count in COPD severity with conflicting findings. $\mathrm{CD} 4^{+}$counts below 200 cells $/ \mu \mathrm{L}$ have been associated with low DLCO $[12,23,24]$ and radiographic emphysema [11, 47•] in PLWH. Older VA data [4] showed that COPD diagnoses by International Classification of Diseases- 9 codes were more likely with lower $\mathrm{CD} 4^{+}$counts, and more recent studies have demonstrated that low $\mathrm{CD}^{+}$counts and a low nadir $\mathrm{CD}^{+}$count are independently associated with a diagnosis of COPD [15 ${ }^{\circ}$. A large study utilizing the AIDS Linked to the Intravenous Experience (ALIVE) cohort found no impact of $\mathrm{CD} 4^{+}$count on the presence of obstruction in cross-sectional analysis [57], but did find that PLWH with $\mathrm{CD}^{+} \leq 200$ cells/ $\mu \mathrm{L}$ had a more rapid decline in FEV1 compared to HIVuninfected individuals [6]. Interestingly, a subanalysis demonstrated that PLWH with $\mathrm{CD}^{+} \geq 350$ cells $/ \mu \mathrm{L}$ and baseline COPD had a fourfold increased risk of acute exacerbations of COPD (AECOPD) compared to HIV-uninfected individuals with COPD [58]. Conversely, longitudinal analysis of the VACS cohort demonstrated increased AECOPD with lower $\mathrm{CD} 4{ }^{+}$counts and high viral load [59]. The reason for these discrepancies may be related to the limitations of crosssectional analysis, as another recent study found no correlation between FEV1 and $\mathrm{CD} 4^{+}$count when looking at only baseline values [17••]. Variation in population demographics, such as the inclusion of only intravenous drug users with heavy smoking exposure in the ALIVE cohort, also prevents findings of individual studies from being generalizable to all 
PLWH. Regardless, these data suggest that individuals with low $\mathrm{CD}^{+}$counts should be considered for spirometry and DLCO screening given the prevalence of abnormal lung function and low DLCO in this population.

\section{HIV Viral Load}

HIV viral load (VL) may also be related to development of COPD. A global epidemiology study found that the prevalence of COPD in PLWH increased with detectable VL [3•]. Similarly, an analysis of COPD diagnosis in a VA cohort found a lower incidence of COPD in individuals with HIV RNA $<400$ copies $/ \mathrm{mL}$ [5]. In a later study conducted by the same group, HIV RNA $>500$ copies $/ \mathrm{mL}$ was associated with lower DLCO in PLWH [23]. Another cross-sectional analysis of PLWH and uninfected participants found no difference in presence of emphysema based on VL or ART use [29]. The largest study to date $(n=1064)$ examining the role of VL on obstructive lung disease occurred in the longitudinal analysis of the ALIVE cohort [6]. While no association was found between ART use and FEV1 decline, participants with a HIV RNA > 75,000 copies $/ \mathrm{mL}$ had a remarkably faster rate of decline in FEV1 (-99.1 mL/year) compared to HIVuninfected participants $(-23.5 \mathrm{~mL} /$ year). These data suggest that VL may modulate the severity of COPD in PLWH.

\section{Antiretroviral Therapy Use}

ART may also impact obstructive lung disease, and studies have had conflicting results of its effect. Two studies have demonstrated that the use of ART is independently associated with airway obstruction in PLWH. These studies have postulated that the mechanism responsible for this relationship may be either a type of immune reconstitution inflammatory syndrome or initiation of autoimmunity within the lungs $[12,13]$. One study was limited by the use of only prebronchodilator spirometry with small absolute differences in FEV1/FVC ratios identified between those receiving and not receiving ART [13], and both were single-center and cross-sectional in nature. In a much larger analysis of the effects of ART use on longitudinal lung function, Kunisaki et al. randomized PLWH at 80 sites in 20 high-income and low-to-middle-income countries to immediate $(n=518)$ versus deferred $(n=508)$ initiation of ART [21•]. Follow-up with annual spirometry was conducted regularly for a median of 2 years. The authors found no difference in rate of FEV1 decline between the two arms, regardless of smoking status. This study was limited by a short period of follow-up due to a mandate for early termination when the parent study showed a clear reduction in AIDS events in the immediate ART arm. It may have also differed from prior work in including individuals with relatively high nadir $\mathrm{CD} 4^{+}$cell counts.
Despite the numerous studies investigating the role of HIVspecific factors on lung function in PLWH, the impact of $\mathrm{CD}^{+}{ }^{+} \mathrm{T}$ cell count, viral load, and ART use remains unclear. Many of the studies discussed above are single center and observational, which limits the strength of the conclusions drawn. Discrepancies may also be due to confounders impacting lung function decline that cannot be controlled for in an observational analysis, e.g., self-efficacy and access to care. The exact degree and timing of immune reconstitution with ART may also influence pulmonary function. Further studies are necessary to better understand how immune activation in the setting of ART use in PLWH affects lung function.

\section{Residual HIV in the Lung}

Despite the success of ART in promoting $\mathrm{CD}^{+} \mathrm{T}$ cell reconstitution, HIV remains latent in PLWH and may influence the long-term pulmonary complications seen in PLWH even with optimal HIV control. While the evidence to-date is conflicting, alveolar macrophages have been theorized to serve as a reservoir for HIV infection although it is difficult to distinguish a viral reservoir in macrophages versus detection of virus in $\mathrm{CD}^{+}$cells that have been ingested by macrophages. One study detected the presence of HIV proviral deoxyribonucleic acid (DNA) in alveolar macrophages from $70 \%$ of healthy PLWH on ART despite undetectable plasma viral loads [60]. The presence of this proviral DNA was associated with impaired alveolar macrophage bacterial phagocytic capacity, which may predispose individuals to infection and chronic inflammation. A recent study using in vitro methods to expose alveolar macrophages from PLWH on ART with undetectable VL to pneumococcal infection found a selective defect in delayed pneumococcal killing by macrophages. The study also found evidence for ongoing viral replication in the alveolar macrophages and bronchoalveolar lavage (BAL) samples of some individuals on ART, suggesting that HIV persists in lung alveolar macrophages despite long-term ART use [61]. However, another study that isolated lymphoid-tissue resident macrophages from chronically simian immunodeficiency virus (SIV)-infected Asian macaques treated with ART found that the viral DNA present in macrophages was not replication-competent [62]. A more recent study of SIV-infected nonhuman primates compared viral DNA levels in $\mathrm{CD}^{+}{ }^{+} \mathrm{T}$-cells collected from the peripheral blood, spleen, and mesenteric lymph nodes of monkeys both on ART and naive to ART [63]. The authors found that all populations of $\mathrm{CD}^{+}{ }^{+} \mathrm{T}$ cells contained viral DNA and no subset of cells was more or less frequently infected by the virus, contradicting the theory that certain tissues harbor HIV DNA more than others. Additional research is needed to further investigate the presence and significance of latent 
virus in various tissues, including the lung and how the virus might relate to lung function.

\section{Lung Microbiome in HIV}

Microbial colonization of the airways in PLWH may also contribute to COPD pathogenesis. The lower respiratory tract in humans was historically considered to be sterile, but recent data have demonstrated the existence of lung microbiome that may contribute to states of health and disease. The lung microbiome is influenced by microbial immigration, regional growth conditions, and microbial elimination [64]. It is hypothesized that the development of lung disease is related to changes in regional growth conditions, which can alter the lung microbiome. The lung microbiota can be influenced by not only bacteria, but also viruses and fungi. Longitudinal analysis of the lung microbiome in humans has been challenging due to the invasive nature of specimen collection via bronchoscopy or lung biopsy. As such, most data are limited to cross-sectional analyses. The influence of lung microbiota on lung function might be important given the residual deficits in lung immunity seen even in individuals with treated HIV and the susceptibility of PLWH to bacterial infections. Inflammation and changes in host response to lung dysbiosis as well as alterations in microbial metabolic products may result from changes in the lung microbiome and lead to or exacerbate lung disease.

Several studies have examined the community distribution of microbes in the oral cavity and airways of PLWH. One cross-sectional analysis of healthy PLWH in London (median $\mathrm{CD}^{+} 609$ cells $/ \mu \mathrm{L} ; 84 \%$ with $\mathrm{VL}<40$ copies $/ \mathrm{mL}$ ) identified common bacterial pneumonia pathogens in the sputum of $43 \%$ of participants using quantitative polymerase chain reaction (PCR), with the majority of colonization attributed to Streptococcus pneumoniae [65]. Higher bacterial loads were found in participants with increased respiratory symptoms, yet no association was found with lung function based on spirometry. The rates of bacterial isolation in PLWH were similar to those seen in HIV-uninfected individuals with COPD, where bacterial colonization is associated with increased exacerbation frequency and long-term lung function decline.

The Lung HIV Microbiome Project, a multicenter US study, sequenced the microbiome of oral washes and BAL of HIV-uninfected individuals and healthy PLWH [66]. There were significant differences in oral microbial populations between HIV-uninfected participants and PLWH, in addition to between PLWH on ART and those naive to ART. Surprisingly, there were no differences in microbial diversity in the lung between HIV-uninfected individuals and PLWH either with or without ART. A more recent longitudinal study comparing the BAL of PLWH to uninfected controls found an altered lung microbiota in individuals with more advanced HIV prior to initiation of ART with decreased alpha diversity (species richness within a sample) and greater beta diversity (community diversity between samples) compared to controls [67]. These differences were reduced over time after initiation of ART but did not return to normal at 3 years after ART initiation.

One finding that has emerged from recent studies is that the bacterial microbiome of the lung in HIV contains the bacteria, Trophyrema whipplei. T. whipplei is a rod-shaped gram-positive bacterium most commonly found in the human intestine [68]. A study of the airway microbiome of nonhuman primates before and after infection with simian HIV (SHIV) demonstrated that the BAL of several monkeys was dominated by T. whipplei [69•]. T. whipplei has been identified in the human lung microbiome as well, with one study detecting greater prevalence in asymptomatic PLWH versus HIVuninfected individuals [70]. The presence of T. whipplei has not been correlated to the development of obstructive lung disease in either monkeys [69•] or humans [71], and the organism is not associated with increased inflammation [71]. The function of $T$. whipplei in the healthy lung has not been established.

The fungal microbiome, or mycobiome, may also be related to lung function in PLWH. Fungi are important allergens and induce inflammation upon contact with airway epithelial cells [72]. Pneumocystis jirovecii, in particular, is known to be a frequent colonizer in both COPD and HIV [73, 74]. A prospective cohort study of PLWH in whom sputum and oral washes were collected demonstrated the association between Pneumocystis colonization and airflow obstruction, independent of smoking history [75]. Those colonized with Pneumocystis also had higher levels of sputum matrix-metalloproteinase-12, which has been identified as contributing to emphysema in cigarettesmoke exposed mice and may play a role in airway remodeling in individuals with COPD [76, 77]. In a recent prospective analysis of oral and respiratory mycobiome in PLWH and uninfected participants, Cui et al. detected fungal communities in BAL samples that differed significantly by HIV status and presence of COPD. Pneumocystis was the most common fungus enriched in BAL samples of both PLWH and those with COPD [78]. Causation between presence of Pneumocystis and obstruction was suggested in a nonhuman primate model of HIV infection in which Shipley et al. demonstrated that SHIV-infected monkeys colonized with Pneumocystis developed progressive obstructive lung disease [79]. BAL samples from these monkeys demonstrated increased levels of Th-2 cytokines and inflammatory mediators, suggesting that Pneumocystis may be a risk factor for development of HIV-associated COPD; however, treatment of Pneumocystis with trimethoprim-sulfamethoxazole in nonhuman primates infected with SHIV and colonized with Pneumocystis did not improve lung function [80]. This lack of response to 
treatment may be due to the importance of other fungi in the lung community or irreversible structural damage of the lung parenchyma associated with Pneumocystis that occurs early after colonization. If the latter is true, efforts at expanding Pneumocystis vaccine development may be helpful in preventing Pneumocystis colonization and the development of obstructive lung disease in PLWH.

\section{Therapies for COPD in HIV}

Appropriate therapies for COPD specific to PLWH are unknown. While inhaled corticosteroids, long-acting beta agonists, and long-acting muscarinic antagonists remain the staple of treatment for uninfected individuals with COPD, trials investigating these therapies have largely excluded PLWH. Inhaled corticosteroids, in particular, should be used with caution in PLWH due to an interaction between inhaled fluticasone and ritonavir that can cause adrenal suppression [81], along with the known increased risk of bacterial pneumonia [82] and TB [83] in a population already at high risk for pulmonary infections. Effects of alternate inhaled steroid formulations in PLWH taking protease inhibitors have not been well-investigated. Only one study to-date has investigated COPD treatment specifically in PLWH. A small randomized controlled pilot study of daily rosuvastatin found that the group receiving rosuvastatin had no decline in FEV1 and an increase in DLCO over 24 weeks with rosuvastatin, though comparisons of change between the two groups did not reach significance [27]. While the anti-inflammatory properties of statins may be responsible for a reduction in lung function decline, larger studies are needed to confirm these findings. Until then, standard COPD therapies will continue to be recommended for PLWH.

\section{Conclusions}

COPD creates a significant burden in PLWH, with onset and progression of disease occurring at younger ages than in the HIV-uninfected population. PLWH demonstrate decreased baseline FEV1 and FVC compared to uninfected smokers, in addition to increased diffusion impairment and radiographic emphysema. PLWH with COPD also experience multiple extrapulmonary comorbidities, including frailty, osteoporosis, cardiovascular disease, and pulmonary hypertension. These COPD phenotypes are associated with increased mortality in PLWH, thus great potential impact exists for identifying pulmonary and cardiovascular complications of HIV at an earlier stage. Multiple HIVspecific factors likely contribute to the pathogenesis of obstructive lung disease in PLWH, with emerging data suggesting a significant role for an altered lung microbiome as well. The most striking modifiable risk factor for obstructive lung disease remains tobacco use. Smoking cessation clearly reduces mortality and needs to be emphasized for all PLWH. Close monitoring of spirometry and DLCO in HIV-infected smokers may identify early pulmonary disease and help encourage efforts at smoking cessation.

\section{Compliance with Ethical Standards}

Conflict of Interest Deepti Singhvi declares that she has no conflict of interest. VA.

Jessica Bon declares that she has received grants from the NIH and the

Alison Morris has received grants from the NIH and Gilead.

Human and Animal Rights and Informed Consent All reported studies/ experiments with human or animal subjects performed by the authors have been previously published and complied with all applicable ethical standards (including the Helsinki declaration and its amendments, institutional/national research committee standards, and international/national/institutional guidelines).

\section{References}

Papers of particular interest, published recently, have been highlighted as:

- Of importance

-• Of major importance

1. Global HIV \& AIDS statistics — 2018 fact sheet/UNAIDS. http:// www.unaids.org/en/resources/fact-sheet. Accessed 23 Feb 2019.

2. Maitre T, Cottenet J, Beltramo G, Georges M, Blot M, Piroth L, et al. Increasing burden of noninfectious lung disease in persons living with HIV: a 7-year study using the French nationwide hospital administrative database. Eur Respir J. 2018;52:1800359. https://doi.org/10.1183/13993003.00359-2018.

3. Bigna JJ, Kenne AM, Asangbeh SL, Sibetcheu AT. Prevalence of chronic obstructive pulmonary disease in the global population with HIV: a systematic review and meta-analysis. Lancet Glob Health. 2018;6:e193-202. This study established the global prevalence of COPD in PLWH to be $10.6 \%$, with a persistent association between COPD and HIV even when controlling for tobacco use. A higher prevalence was seen in those with higher income, more tobacco use, detectable viral load, and from European countries.

4. Crothers K, Butt AA, Gibert CL, Rodriguez-Barradas MC, Crystal $\mathrm{S}$, Justice AC, et al. Increased COPD among HIV-positive compared to HIV-negative veterans. Chest. 2006;130:1326-33.

5. Crothers K, Huang L, Goulet JL, Goetz MB, Brown ST, RodriguezBarradas MC, et al. HIV infection and risk for incident pulmonary diseases in the combination antiretroviral therapy era. Am J Respir Crit Care Med. 2011;183:388-95.

6. Drummond MB, Merlo CA, Astemborski J, Kalmin MM, Kisalu A, Mcdyer JF, et al. The effect of HIV infection on longitudinal lung function decline among IDUs: a prospective cohort. AIDS. 2013;27:1303-11. 
7. Drummond MB, Kunisaki KM, Huang L. Obstructive lung diseases in HIV: a clinical review and identification of key future research needs. Semin Respir Crit Care Med. 2016;37:277-88.

8. Makinson A, Hayot M, Eymard-Duvernay S, Ribet C, Raffi F, Pialoux G, et al. HIV is associated with airway obstruction: a matched controlled study. AIDS. 2018;32:227-32.

9. Global Initiative for Chronic Obstructive Lung Disease. Global strategy for the diagnosis, management, and prevention of chronic obstructive pulmonary disease: 2019 report. http://www.goldcopd. org. Accessed 22 Feb 2019.

10. Han MK, Agusti A, Calverley PM, et al. Chronic obstructive pulmonary disease phenotypes: the future of COPD. Am J Respir Crit Care Med. 2010;182:598-604.

11. Sampériz G, Guerrero D, López M, Valera JL, Iglesias A, Ríos Á, et al. Prevalence of and risk factors for pulmonary abnormalities in HIV-infected patients treated with antiretroviral therapy. HIV Med. 2014;15:321-9.

12. Gingo MR, George MP, Kessinger CJ, Lucht L, Rissler B, Weinman R, et al. Pulmonary function abnormalities in HIVinfected patients during the current antiretroviral therapy era. Am J Respir Crit Care Med. 2010;182:790-6.

13. George MP, Kannass M, Huang L, Sciurba FC, Morris A. Respiratory symptoms and airway obstruction in HIV-infected subjects in the HAART era. PLoS One. 2009;4:e6328.

14. Ronit A, Lundgren J, Afzal S, Benfield T, Roen A, Mocroft A, et al. Airflow limitation in people living with HIV and matched uninfected controls. Thorax. 2018;73:431-8.

15. Risso K, Guillouet-de-Salvador F, Valerio L, Puglièse P, Naqvi A, Durant $\mathrm{J}$, et al. COPD in HIV-infected patients: CD4 cell count highly correlated. PLoS One. 2017;12:e0169359. This study, a single center cross-sectional analysis of HIV-infected patients in France, found that low CD4 cell count and a low nadir CD4 cell count are independently associated with a diagnosis of COPD.

16. Hirani A, Cavallazzi R, Vasu T, Pachinburavan M, Kraft WK, Leiby B, et al. Prevalence of obstructive lung disease in HIV population: a cross sectional study. Respir Med. 2011;105:1655-61.

17.• Li Y, Nouraie SM, Kessinger C, Weinman R, Huang L, Greenblatt $\mathrm{RM}$, et al. Factors associated with progression of lung function abnormalities in HIV-infected individuals. J Acquir Immune Defic Syndr. 2018;79:501-9. This study demonstrated decreased DLCO in $79 \%$ of PLWH with faster rates of FEV1 decline in patients who were male with higher GOLD stage and older age. There was no difference in baseline FEV1 based on $\mathrm{CD4}^{+}$ count, viral load, or ART use.

18. Drummond MB, Kirk GD, Astemborski J, McCormack MC, Marshall MM, Mehta SH, et al. Prevalence and risk factors for unrecognized obstructive lung disease among urban drug users. Int J Chron Obstruct Pulmon Dis. 2011;6:89-95.

19. Vestbo J, Edwards LD, Scanlon PD, Yates JC, Agusti A, Bakke P, et al. Changes in forced expiratory volume in 1 second over time in COPD. N Engl J Med. 2011;365:1184-92.

20. Casanova C, de Torres JP, Aguirre-Jaíme A, Pinto-Plata V, Marin $\mathrm{JM}$, Cordoba E, et al. The progression of chronic obstructive pulmonary disease is heterogeneous: the experience of the BODE cohort. Am J Respir Crit Care Med. 2011;184:1015-21.

21. MacDonald DM, Melzer AC, Collins G, et al. Smoking and accelerated lung function decline in HIV-positive individuals: a secondary analysis of the START pulmonary substudy. J Acquir Immune Defic Syndr. 2018;79:e85-92. In this secondary analysis of the START pulmonary substudy, the authors found a faster rate of annual decline in FEV1 in HIV-infected smokers compared to nonsmokers.

22.• Kunisaki KM, Niewoehner DE, Collins G, Aagaard B, Atako NB, Bakowska E, et al. Pulmonary effects of immediate versus deferred antiretroviral therapy in HIV-positive individuals: a nested substudy within the multicentre, international, randomised, controlled Strategic Timing of Antiretroviral Treatment (START) trial. Lancet Respir Med. 2016;4:980-9. This was a pulmonary substudy of a randomized controlled trial in which participants with HIV infection were randomized to immediate initiation of ART versus deferred until $\mathrm{CD}^{+}$cell count $<350$ cells $/ \mu \mathrm{l}$. No difference was found between these groups in the rate of annual FEV1 decline, suggesting that the use of ART does not impact lung function decline.

23. Crothers K, McGinnis K, Kleerup E, Wongtrakool C, Hoo GS, Kim $\mathrm{J}$, et al. HIV infection is associated with reduced pulmonary diffusing capacity. J Acquir Immune Defic Syndr. 2013;64:271-8.

24. Fitzpatrick ME, Gingo MR, Kessinger C, Lucht L, Kleerup E, Greenblatt RM, et al. HIV infection is associated with diffusing capacity impairment in women. J Acquir Immune Defic Syndr. 2013;64:284-8.

25. Gingo MR, He J, Wittman C, Fuhrman C, Leader JK, Kessinger C, et al. Contributors to diffusion impairment in HIV-infected persons. Eur Respir J. 2014;43:195-203.

26. Neas LM, Schwartz J. The determinants of pulmonary diffusing capacity in a national sample of U.S. adults. Am J Respir Crit Care Med. 1996;153:656-64.

27. Morris A, Fitzpatrick M, Bertolet M, Qin S, Kingsley L, Leo N, et al. Use of rosuvastatin in HIV-associated chronic obstructive pulmonary disease. AIDS. 2017;31:539-44.

28. Diaz PT, King MA, Pacht ER, Wewers MD, Gadek JE, Nagaraja $\mathrm{HN}$, et al. Increased susceptibility to pulmonary emphysema among HIV-seropositive smokers. Ann Intern Med. 2000;132:369-72.

29. Attia EF, Akgün KM, Wongtrakool C, Goetz MB, RodriguezBarradas MC, Rimland D, et al. Increased risk of radiographic emphysema in HIV is associated with elevated soluble CD14 and nadir CD4. Chest. 2014;146:1543-53.

30. Leader JK, Crothers K, Huang L, King MA, Morris A, Thompson $\mathrm{BW}$, et al. Risk factors associated with quantitative evidence of lung emphysema and fibrosis in an HIV-infected cohort. J Acquir Immune Defic Syndr. 2016;71:420-7.

31.• Triplette M, Attia EF, Akgün KM, Soo Hoo GW, Freiberg MS, Butt $\mathrm{AA}$, et al. A low peripheral blood $\mathrm{CD} 4 / \mathrm{CD} 8$ ratio is associated with pulmonary emphysema in HIV. PLoS One. 2017;12:e0170857. In this study, the authors found that a low peripheral CD4:CD8 ratio is associated with radiographic emphysema and low DLCO in PLWH, suggesting that this blood test may be able to be used as a marker of emphysema in PLWH.

32.• Leung JM, Malagoli A, Santoro A, Besutti G, Ligabue G, Scaglioni $\mathrm{R}$, et al. Emphysema distribution and diffusion capacity predict emphysema progression in human immunodeficiency virus infection. PLoS One. 2016;11:e0167247. This study demonstrated that emphysema progression can be predicted based on radiographic emphysema distribution and DLCO values. PLWH who were more likely to have emphysema progression had higher baseline emphysema score and greater smoking exposure history.

33. Guaraldi G, Besutti G, Scaglioni R, Santoro A, Zona S, Guido L, et al. The burden of image based emphysema and bronchiolitis in HIV-infected individuals on antiretroviral therapy. PLoS One. 2014;9:e109027.

34. Mannino DM, Thorn D, Swensen A, Holguin F. Prevalence and outcomes of diabetes, hypertension and cardiovascular disease in COPD. Eur Respir J. 2008;32:962-9.

35. Bon J, Fuhrman CR, Weissfeld JL, Duncan SR, Branch RA, Chang $\mathrm{CCH}$, et al. Radiographic emphysema predicts low bone mineral density in a tobacco-exposed cohort. Am J Respir Crit Care Med. 2011;183:885-90.

36. Hegerl U, Mergl R. Depression and suicidality in COPD: understandable reaction or independent disorders? Eur Respir J. 2014;44: 734-43. 
37. Bloch M. Frailty in people living with HIV. AIDS Res Ther. 2018;15:19.

38. Kooij KW, Wit FWNM, Schouten J, van der Valk M, Godfried MH, Stolte IG, et al. HIV infection is independently associated with frailty in middle-aged HIV type 1-infected individuals compared with similar but uninfected controls. AIDS. 2016;30:241-50.

39. Akgün KM, Tate JP, Oursler KK, Crystal S, Leaf DA, Womack JA, et al. Association of chronic obstructive pulmonary disease with frailty measurements in HIV-infected and uninfected veterans. AIDS. 2016;30:2185-93. This study identified that while COPD is strongly associated with frailty in both HIV-infected and HIV-uninfected individuals, there is a stronger association in HIV-infected individuals. COPD is an independent risk factor for frailty in PLWH.

40. Sin DD, Man JP, Man SFP. The risk of osteoporosis in Caucasian men and women with obstructive airways disease. Am J Med. 2003;114:10-4.

41. Bonjoch A, Figueras M, Estany C, Perez-Alvarez N, Rosales J, del Rio L, et al. High prevalence of and progression to low bone mineral density in HIV-infected patients: a longitudinal cohort study. AIDS. 2010;24:2827-33.

42. Guaraldi G, Orlando G, Zona S, Menozzi M, Carli F, Garlassi E, et al. Premature age-related comorbidities among HIV-infected persons compared with the general population. Clin Infect Dis. 2011;53:1120-6.

43. Petraglia A, Leader JK, Gingo M, Fitzpatrick M, Ries J, Kessinger $\mathrm{C}$, et al. Emphysema is associated with thoracic vertebral bone attenuation on chest CT scan in HIV-infected individuals. PLoS One. 2017;12:e0176719. The authors in this study measured thoracic vertebral bone attenuation as a surrogate for bone mineral density on CT chest imaging in PLWH. They found that greater emphysema is independently associated with lower bone mineral density in PLWH and the use of ART further reduces this bone mineral density.

44. Hasse B, Ledergerber B, Furrer H, Battegay M, Hirschel B, Cavassini $\mathrm{M}$, et al. Morbidity and aging in HIV-infected persons: the Swiss HIV cohort study. Clin Infect Dis. 2011;53:1130-9.

45. Alwan A. (2011). Burden: mortality, morbidity, and risk factors. Global status report on noncommunicable diseases (pp. 9-31). Geneva, Switzerland: World Health Organization.

46. Chandra D, Gupta A, Leader JK, Fitzpatrick M, Kingsley LA, Kleerup E, et al. Assessment of coronary artery calcium by chest CT compared with EKG-gated cardiac CT in the multicenter AIDS cohort study. PLoS One. 2017;12:e0176557.

47. Besutti G, Raggi P, Zona S, Scaglioni R, Santoro A, Orlando G, et al. Independent association of subclinical coronary artery disease and emphysema in HIV-infected patients. HIV Med. 2016;17:17887. This study scored emphysema and coronary artery calcium on CT chest radiographs from PLWH and found the presence of emphysema is independently associated with a positive coronary artery calcium score. Radiographic emphysema was also associated with a $\mathrm{CD4}{ }^{+}$count nadir $<200$ cells $/ \mu \mathrm{l}$.

48. Morris A, Gingo MR, George MP, Lucht L, Kessinger C, Singh V, et al. Cardiopulmonary function in individuals with HIV infection in the antiretroviral therapy era. AIDS. 2012;26:731-40.

49. Brittain EL, Duncan MS, Chang J, Patterson OV, DuVall SL, Brandt CA, et al. Increased echocardiographic pulmonary pressure in HIV-infected and -uninfected individuals in the veterans aging cohort study. Am J Respir Crit Care Med. 2018;197:923-32.

50.• Triplette M, Justice A, Attia EF, Tate J, Brown ST, Goetz MB, et al. Markers of chronic obstructive pulmonary disease are associated with mortality in people living with HIV. AIDS. 2018;32:487-93. This study demonstrated that airflow obstruction, DLCO, and emphysema are associated with increased mortality, independent of smoking exposure, in PLWH.
51.• Gingo MR, Nouraie M, Kessinger CJ, Greenblatt RM, Huang L, Kleerup EC, et al. Decreased lung function and all-cause mortality in HIV-infected individuals. Ann Am Thorac Soc. 2018;15:192-9. This study reported that both obstruction (FEV1/FVC $<0.7)$ and diffusion impairment (DLCO $<60 \%$ ) are associated with increased mortality in PLWH. The decrease in the KaplanMeier curve occurs earlier in time for diffusion impairment, suggesting that this may be an earlier marker of mortality.

52. Shirley DK, Kaner RJ, Glesby MJ. Effects of smoking on nonAIDS-related morbidity in HIV-infected patients. Clin Infect Dis. 2013;57:275-82.

53. Popescu I, Drummond MB, Gama L, Coon T, Merlo CA, Wise RA, et al. Activation-induced cell death drives profound lung CD4(+) Tcell depletion in HIV-associated chronic obstructive pulmonary disease. Am J Respir Crit Care Med. 2014;190:744-55.

54. Brune KA, Ferreira F, Mandke P, Chau E, Aggarwal NR, D’Alessio FR, et al. HIV impairs lung epithelial integrity and enters the epithelium to promote chronic lung inflammation. PLoS One. 2016;11:e0149679.

55. Rosen MJ, Lou Y, Kvale PA, Rao AV, Jordan MC, Miller A, et al. Pulmonary function tests in HIV-infected patients without AIDS. Pulmonary Complications of HIV Infection Study Group. Am J Respir Crit Care Med. 1995;152:738-45.

56. Kuhlman JE, Knowles MC, Fishman EK, Siegelman SS. Premature bullous pulmonary damage in AIDS: CT diagnosis. Radiology. 1989; 173:23-6.

57. Drummond MB, Kirk GD, Astemborski J, Marshall MM, Mehta $\mathrm{SH}, \mathrm{McDyer} J \mathrm{~F}$, et al. Association between obstructive lung disease and markers of HIV infection in a high-risk cohort. Thorax. 2012;67:309-14.

58. Lambert AA, Kirk GD, Astemborski J, Mehta SH, Wise RA, Drummond MB. HIV infection is associated with increased risk for acute exacerbation of COPD. J Acquir Immune Defic Syndr. 2015;69:68-74.

59. Depp TB, McGinnis KA, Kraemer K, Akgün KM, Edelman EJ, Fiellin DA, et al. Risk factors associated with acute exacerbation of chronic obstructive pulmonary disease in HIV-infected and uninfected patients. AIDS. 2016;30:455-63.

60. Cribbs SK, Lennox J, Caliendo AM, Brown LA, Guidot DM. Healthy HIV-1-infected individuals on highly active antiretroviral therapy harbor HIV-1 in their alveolar macrophages. AIDS Res Hum Retrovir. 2015;31:64-70.

61. Collini PJ, Bewley MA, Mohasin M, Marriott HM, Miller RF, Geretti AM, et al. HIV gp120 in the lungs of antiretroviral therapy-treated individuals impairs alveolar macrophage responses to pneumococci. Am J Respir Crit Care Med. 2018;197:1604-15.

62. DiNapoli SR, Ortiz AM, Wu F, Matsuda K, Twigg HL, Hirsch VM, et al. Tissue-resident macrophages can contain replicationcompetent virus in antiretroviral-naive, SIV-infected Asian macaques. JCI Insight. 2017;2:e91214.

63. Lai S, Starke CE, Flynn JK, Vinton CL, Ortiz AM, Mudd JC, et al. SIV infects functionally polarized memory CD4 T cells equivalently in vivo. J Virol. 2019;93. https://doi.org/10.1128/JVI.02163-18.

64. Dickson RP, Erb-Downward JR, Martinez FJ, Huffnagle GB. The microbiome and the respiratory tract. Annu Rev Physiol. 2016;78: 481-504.

65. Nimmo C, Capocci S, Honeyborne I, Brown J, Sewell J, Thurston $\mathrm{S}$, et al. Airway bacteria and respiratory symptoms are common in ambulatory HIV-positive UK adults: TABLE 1. Eur Respir J. 2015;46:1208-11.

66. Beck JM, Schloss PD, Venkataraman A, Twigg H 3rd, Jablonski KA, Bushman FD, et al. Multicenter comparison of lung and oral microbiomes of HIV-infected and HIV-uninfected individuals. Am J Respir Crit Care Med. 2015;192:1335-44. 
67. Twigg HL, Knox KS, Zhou J, et al. Effect of advanced HIV infection on the respiratory microbiome. Am J Respir Crit Care Med. 2016;194:226-35.

68. Dolmans RAV, Boel CHE, Lacle MM, Kusters JG. Clinical manifestations, treatment, and diagnosis of Tropheryma whipplei infections. Clin Microbiol Rev. 2017;30:529-55.

69. Morris A, Paulson JN, Talukder H, Tipton L, Kling H, Cui L, et al. Longitudinal analysis of the lung microbiota of cynomolgous macaques during long-term SHIV infection. Microbiome. 2016;4:38. In a study of microbiome analysis from serial bronchoalveolar lavage samples in nonhuman primates before and after infection with SHIV, half of the monkeys developed COPD over longitudinal follow-up and tended to have more oral bacteria in their BAL microbiota. There was no relationship between the presence of Tropheryma whipplei in BAL and the development of COPD.

70. Lozupone C, Cota-Gomez A, Palmer BE, Linderman DJ, Charlson ES, Sodergren E, et al. Widespread colonization of the lung by Tropheryma whipplei in HIV infection. Am J Respir Crit Care Med. 2013;187:1110-7.

71. Qin S, Clausen E, Nouraie SM, Kingsley L, McMahon D, Kleerup E, et al. Tropheryma whipplei colonization in HIV-infected individuals is not associated with lung function or inflammation. PLoS One. 2018;13:e0205065.

72. Roy RM, Klein BS. Fungal glycan interactions with epithelial cells in allergic airway disease. Curr Opin Microbiol. 2013;16:404-8.

73. Morris A, Sciurba FC, Lebedeva IP, Githaiga A, Elliott WM, Hogg JC, et al. Association of chronic obstructive pulmonary disease severity and Pneumocystis colonization. Am J Respir Crit Care Med. 2004;170:408-13

74. Morris A, Kingsley LA, Groner G, Lebedeva IP, Beard CB, Norris KA. Prevalence and clinical predictors of Pneumocystis colonization among HIV-infected men. AIDS. 2004;18:793-8.

75. Morris A, Alexander T, Radhi S, Lucht L, Sciurba FC, Kolls JK, et al. Airway obstruction is increased in Pneumocystis-colonized human immunodeficiency virus-infected outpatients. J Clin Microbiol. 2009;47:3773-6.
76. Hautamaki RD, Kobayashi DK, Senior RM, Shapiro SD. Requirement for macrophage elastase for cigarette smokeinduced emphysema in mice. Science. 1997;277:2002-4.

77. Sato M, Hirayama S, Lara-Guerra H, Anraku M, Waddell TK, Liu $\mathrm{M}$, et al. MMP-dependent migration of extrapulmonary myofibroblast progenitors contributing to posttransplant airway fibrosis in the lung. Am J Transplant. 2009;9:1027-36.

78. Cui L, Lucht L, Tipton L, Rogers MB, Fitch A, Kessinger C, et al. Topographic diversity of the respiratory tract mycobiome and alteration in HIV and lung disease. Am J Respir Crit Care Med. 2015;191:932-42.

79. Shipley TW, Kling HM, Morris A, Patil S, Kristoff J, Guyach SE, et al. Persistent Pneumocystis colonization leads to the development of chronic obstructive pulmonary disease in a nonhuman primate model of AIDS. J Infect Dis. 2010;202:302-12.

80. Kling HM, Shipley TW, Guyach S, Tarantelli R, Morris A, Norris KA. Trimethoprim-sulfamethoxazole treatment does not reverse obstructive pulmonary changes in Pneumocystis-colonized nonhuman primates with SHIV infection. J Acquir Immune Defic Syndr. 2014;65:381-9.

81. Foisy MM, Yakiwchuk EMK, Chiu I, Singh AE. Adrenal suppression and Cushing's syndrome secondary to an interaction between ritonavir and fluticasone: a review of the literature. HIV Med. 2008;9:389-96.

82. Crim C, Calverley PMA, Anderson JA, Celli B, Ferguson GT, Jenkins $\mathrm{C}$, et al. Pneumonia risk in COPD patients receiving inhaled corticosteroids alone or in combination: TORCH study results. Eur Respir J. 2009;34:641-7.

83. Brassard P, Suissa S, Kezouh A, Ernst P. Inhaled corticosteroids and risk of tuberculosis in patients with respiratory diseases. Am J Respir Crit Care Med. 2011;183:675-8.

Publisher's Note Springer Nature remains neutral with regard to jurisdictional claims in published maps and institutional affiliations. 